\title{
« La publication des lois par des parlementaires de province ». L'exemple bordelais durant le règne de François $I^{\text {er }}(1515-1547)$
}

Elise Frêlon

Elise Frêlon : Elise Frêlon est Maitre de conférences en Histoire du Droit à l'Université de Poitiers. Elle est l'auteur de l'ouvrage Le Parlement de Bordeaux et la "loi" (1451-1547), Paris, De Boccard, Collection Romanité et Modernité du Droit, 2011; ainsi que de nombreux articles et communications en histoire du Droit.

DOI: $10.25518 / 1370-2262.424$

\section{Résumé :}

L'historien du droit a pour fonction de remonter dans le passé afin de retrouver l'origine de son propre présent. La présente réflexion se conçoit de façon synchronique et, comme telle, est focalisée sur l'instant $t$ du règne de François $\mathrm{I}^{\mathrm{er}}$. A cette date, le roi ne légifère pas. Dans ses lettres royaux, il ordonne. Et à ses parlementaires incombe une publication desdites ordonnances. Autrement dit, littéralement, si le roi ne rédige pas encore de « loi », les parlementaires n'en sont pas moins tenus à une «publication » de ses ordonnances (non sans altération - moderne - des termes anciens de lex et publicatio). Une étude juridique est donc possible : celle de la promulgation des lettres royaux par des parlementaires en province, en l'espèce en Guyenne. Évoquer successivement ses enjeux et difficultés (I), temps et lieux (II) acteurs et méthodes (III) permet de préciser ce postulat d'une législation entendue comme un moyen de communication entre gouvernés et gouvernants.

Mots-clés : Parlements - royaume de France - publication - lettres royaux

\section{Introduction. Depuis quand légiférer est-ce communiquer?}

«Quand légiférer c'est communiquer », telle est la problématique que Nicolas Simon a soumise à une réflexion collective. Qu'il en soit cordialement remercié et qu'il trouve en ces lignes une réponse individuelle qui - cela va sans dire - n'engage que son auteur.

Légiférer et publier (à défaut de communiquer stricto sensu1) sont, dans le droit français le plus positif qui soit, inexorablement liés : la publication confère à la loi le terminus a quo de son entrée en vigueur $\underline{2}$, une vigueur qui est celle de sa force obligatoire. De sorte que la loi n'obligerait pas le peuple tant qu'elle ne serait pas portée à sa connaissance. Fort de cette acception actuelle $\underline{3}$, l'historien du droit a pour vocation de remonter aussi haut que faire se peut dans le passé aux principes $\underline{4}$ - afin de retrouver l'origine de son propre présent. Dans cette quête à rebours de l'instant t, la chronologie le préserve de tout anachronisme $\underline{5}$. En toute rigueur académique, quatre temps (qui ont chacun leur champ lexical propreb) ponctuent cette chronologie que sont l'Antiquité, le Moyen Age, les Temps Modernes et l'Époque Contemporaine - dans cet ordre qu'il faut se garder d'inverser $\underline{7}$ - et dont la succession, seule, désigne l'évolution $\underline{8}$. Or, en matière d'institutions («publiques » a fortiori), et sauf exceptions «germaniques » (franques en particulier), la maior et sanior pars de nos fondements juridiques actuels sont romains $\underline{9}$. Les jurisprudents classiques en effet, ont offert à la postérité, et partant, à leurs successeurs (médiévaux, modernes, contemporains) 
« La publication des lois par des parlementaires de province ». L'exemple bor...

les éléments fondamentaux de toute science juridique.

Ni la loi (lat. lex), ni la publication (lat. publicatio) n'échappent à cette source classique providentiellement compilée par Justinien10. D’hier à aujourd’hui, ces deux catégories dogmatiques que sont la loi et la publication se sont donc maintenues, a priori. Un tel a priori repose sur la continuité terminologique dont bénéficient les langues romanes. Toutefois, si les termes sont saufs (ou quasi par la traduction du latin au français), il en est autrement de leur sens : leurs définitions ont été altérées, trahissant une discontinuité sémantique, méthodologiquement contraire au nominalisme qui attribue à chaque terme une définition, technique et surtout invariable 11.

La mise en regard des définitions d'hier (droit romain classique) et d'aujourd'hui (droit français positif) en atteste :

$\underline{12} ; \underline{13} ; \underline{14} ; \underline{15}$.

\begin{tabular}{|c|c|}
\hline Lex & Loi \\
\hline $\begin{array}{c}\text { «Ce que le peuple Romain constituait, un } \\
\text { magistrat sénatorial l'interrogeant, par } \\
\text { exemple un consul } »^{12} .\end{array}$ & « Texte voté par le Parlement ${ }^{13}$. \\
\hline
\end{tabular}

\begin{tabular}{|c|c|}
\hline Publication (bonorum) ${ }^{14}$ & Publication (de la loi) \\
\hline $\begin{array}{l}\text { Attribution des biens d'un citoyen (singulier) } \\
\text { au peuple (somme plurielle des citoyens). }\end{array}$ & $\begin{array}{l}\text { "Action de porter un acte législatif [...] à la } \\
\text { connaissance du public [...]; mesure de } \\
\text { publicité destinée à rendre l'acte opposable à } \\
\text { tous qui constitue l'une des conditions de } \\
\text { l'entrée en vigueur de l'acte } \aleph^{15} \text {. }\end{array}$ \\
\hline
\end{tabular}

Ces divergences sémantiques de deux mêmes termes juridiques (loi, publication) à deux termes historiques distincts (terminus a quo 533, terminus ad quem 2016) ainsi mises en évidence, il appartient à l’historien de les dater $\underline{16}$. Face à cette ambition, la présente réflexion se conçoit de façon délibérément synchronique17: qu'en est-il de la loi et de sa publication aux Temps Modernes ? Pour tenter d'amorcer une réponse, l'on peut se reporter au fameux Dictionnaire des Ferrière 18 emblématique de cette époque. Les deux termes y sont aisément accessibles :

$\underline{19} ; \underline{20}$.

\begin{tabular}{|c|c|}
\hline Loi & $\begin{array}{c}\text { Publication (des Ordonnances, Édits et } \\
\text { Déclarations) }\end{array}$ \\
\hline $\begin{array}{c}\text { « Constitution ou Ordonnance Générale, qui } \\
\text { vient d'une autorité souveraine [...] avec } \\
\text { injonction d'obéir à sa décision } \aleph^{20}\end{array}$ & $\begin{array}{c}\text { " Lecture qui s'en fait dans les Cours, pour } \\
\text { être connues au Peuple \& être ensuite } \\
\text { exécutées ». }\end{array}$ \\
\hline
\end{tabular}

Cette étape entre les acceptions antiques et actuelles des termes envisagés, dans laquelle s'insèrent opportunément «les Cours », peut être précisée encore et, comme telle, focalisée sur l'instant t du règne de François $\mathrm{I}^{\mathrm{er}}$. En humaniste convaincu, le premier des François établit officiellement une civilisation française 21 (Moderne) distincte de la civilisation latine (des Anciens). Encore que l'innovation linguistique de son règne - l'Ordonnance de Villers Cotterêts $\underline{22}$ - ne soit pas 
populaire : elle n'est pas destinée à tous les sujets du roi ou à tous les habitants du royaume, mais judiciaire 23 : c'est aux magistrats qu'obligation est faite de cesser d'exprimer leurs arrêts en latin. Institutionnellement (et sous la souveraineté du roi) les parlements du royaume, de Paris ou des autres capitales des provinces, sont au sommet de cet ordre judiciaire auquel est destinée l'ordonnance précitée. En cette première moitié d'un $\mathrm{XVI}^{\mathrm{e}}$ siècle de Renaissance, ses membres ne sont-ils pas des acteurs majeurs de la loi et de sa publication?

À ne s'en tenir qu'à l'étude provinciale du seul parlement de Bordeaux (érigé par Charles VII, en 1451 à la faveur de la reconquête du duché d'Aquitaine ou " Guyenne »), ses sources24 dépouillées permettent d'apprécier la réalité normative du temps passé, au regard des actes expédiés par la chancellerie royale et reçus en la cour à toutes fins utiles.

Létude diplomatique de ces actes $\underline{25}$ atteste qu'au moins jusqu'au règne de Henri II, le roi ne légifère pas $\underline{26}$. Dans ses «lettres royaux », il ordonne principalement, et édicte ou déclare accessoirement. Et aux parlementaires incombe une publication desdits ordonnances, édits, déclarations qu'un historien du droit contemporain a pu associer a posteriori sous le qualificatif unique de « lois du roi »27. Autrement dit, et en vertu des sources, au sens actuel - altéré du sens initial - des termes, si le roi ne rédige pas encore de loi, les parlementaires n'en sont pas moins déjà tenus à une publication de ses ordonnances.

Force est de constater que la réalité juridique du populus et de la lex antiques semblent avoir disparu28 du champ institutionnel de la Renaissance $\underline{29}$. En leur lieu et place, le souverain $\underline{30}$ et l'ordonnance, termes tous deux d'origine médiévale, se sont maintenus. La rupture est ainsi déjà consommée entre les Anciens Romains et les Modernes François : les premiers associent au populus la maiestas et la lex, les seconds parant le seul souverain (il s'agit alors du monarque et non plus du peuple) de ces attributs (majesté et loi). À défaut juridique de peuple $\underline{31}$ - et par conséquent de loi (puisque celle-ci, techniquement32, est la constitution de celui-là : non seulement la loi est pour le peuple mais aussi, et indispensablement, elle est du peuple et par le peuple) - la réalité politique de ces Temps, a posteriori qualifiés de Modernes, est celle d'un «public » assujetti à un roi, celle de sujets (libres au demeurant) régis par le premier d'entre eux.

En toute modernité, la publication de la loi n'est rien moins qu'une conséquence de cette ruine politique du «peuple», acteur juridique $\underline{3}$ déchu par le roi et ses légistes en «public » $\underline{34}$, spectateur 35 sociologique. Comme telle, elle consiste dès lors à informer une population de l'existence d'un acte impératif $\underline{36}$ (telle est la loi), acte sur la teneur duquel - précisément - les citoyens $\underline{37}$ (i.e. le peuple) ne sont plus $\underline{38}$ conviés au vote.

Faute de pouvoir, historiquement, tout à fait concevoir de rapport entre légiférer et publier (et a fortiori communiquer) durant le règne de François $\mathrm{I}^{\mathrm{er}}$, une autre étude juridique est possible : celle de la «promulgation $\gg \underline{39}$ ou de la « divulgation $\$ \underline{40}$ des lettres royaux par des parlementaires en province, en l'espèce en Guyenne. Évoquer successivement ses enjeux et difficultés (I), temps et lieux (II), acteurs et méthodes (III) permettra de préciser quelque peu ce postulat de la publication d'une législation 41 " entendue comme un moyen de communication entre gouvernés et gouvernants $\gg \underline{42}$.

\section{Les enjeux et difficultés}

La promulgation des lettres patentes parait être une condition de leur diffusion, mais aucune 
« La publication des lois par des parlementaires de province ». L'exemple bor...

ordonnance, aucun édit ni aucune déclaration d'un monarque français n'a érigé en principe la publication des patentes, petites ou grandes, ni au sein du parlement, ni du reste en aucune juridiction quelle qu'elle soit. Toutefois, c'est spécifiquement l'institution durable d'un parlement à Bordeaux, gardien désigné des actes royaux dans son ressort qui - si ce n'est seule du moins en grande partie - a permis le progrès et l'affirmation de la législation royale dans l'ordre juridique établi.

La connaissance de la pratique suppose la maîtrise de la diplomatique des lettres royaux puisque, parmi les diverses clauses finales, certaines ont - spécifiquement ou incidemment - pour objet la publication. Lorsqu'il s'adresse à ses magistrats, le monarque peut leur mander ou enjoindre de «publier» ses lettres, sans toutefois qu'une telle clause ne soit de style, loin s'en faut. À ces mandements ou injonction de publier sont associés ceux de " préconiser », « cryer », "signiffier », faire « scavoir » ou « notiffier et proclamer » $\underline{43}$.

Quant à la finalité - au moins médiévale 44 - d'une telle formalité : afin que nul n'en puisse prétendre ignorance, elle est plus rarement exprimée encore $\underline{45}$. Reste qu'un enjeu fondamental de cette « cérémonie de l'information » $\underline{46}$ consiste à divulguer la volonté du roi à ses sujets : comme telle la publication est une divulgation $\underline{47}$ ou promulgation $\underline{48}$. Toute promulgation des prescriptions royales est populaire dès lors qu'elle est destinée au public que constituent les sujets en devoir de leur application. À l'époque moderne, la force obligatoire de la loi repose ainsi, non plus sur l'acceptation de celle-ci exprimée par les suffrages du peuple (définition antique), mais sur la connaissance qu'en livre la promulgation (doctrine médiévale $\underline{49}$ ). En d'autres termes, tandis qu'à l'époque antique la force obligatoire de la loi résidait dans son consentement par le peuple, la doctrine médiévale - sur laquelle repose la pratique moderne - lui a substitué sa connaissance. De la sorte, le peuple demeure au cœur de la loi, comme récepteur (en étant gouverné), mais non plus comme émetteur (en étant son propre gouvernant); ce que conforte l'assimilation tronquée $\underline{50}$, elle aussi médiévale, du roy à la loy. La proclamation des patentes est non seulement juridique mais de surcroît hautement symbolique $\underline{1}$. Temps et lieux comme acteurs et méthodes ne sont pas choisis et ritualisés au hasard프. Sous la responsabilité des parlementaires, la publication des actes royaux est donc un moyen de promouvoir la législation royale - tant dans les cours de justice que dans les villes, places et lieux publics du ressort - en même temps que d'affirmer la présence du monarque auprès de ses sujets. Son efficacité peut alors se mesurer aux difficultés que peut soulever sa pratique.

Il appartient aux parlementaires de s'assurer du bon déroulement de la proclamation populaire des actes royaux. La tâche n'est pas exempte d'incidents puisque c'est précisément à cette occasion alors que les prescriptions de leur monarque sont portées à leur connaissance - que les sujets peuvent commencer à manifester certaines résistances. Aussi est-ce essentiellement afin de « tenir en surséance la publication » $\underline{53}$ que se multiplient des « appellations » ou « oppositions » aux actes royaux de la part de tiers lésés par ceux-ci, individuellement ou collectivement.

Les résistances populaires inhérentes aux publications des actes royaux peuvent parfois être fort virulentes. En Guyenne, pays aux vastes frontières maritimes dont la richesse repose en grande partie sur la production saline, c'est essentiellement l'institution de la gabelle par François $\mathrm{I}^{\mathrm{er}}$ qui provoque des incidents violents (coups d'arquebuses tirés de nuit ou insultes) et qui décide les commissaires chargés de la publication de l'« édit du sel » à requérir qu'un conseiller et des huissiers les accompagnent $\underline{54}$. 
Que la proclamation des lettres royaux soit ainsi ponctuellement confiée aux présidents et conseillers, mais surtout aux huissiers du parlement n'a rien d'étonnant dans la mesure où, d'une façon générale, c'est à ces derniers qu'appartient de transmettre aux intéressés les lettres de la chancellerie royale. Toutefois, avant de concevoir dans leur détail les acteurs et les méthodes de la publication des patentes, il faut s'arrêter sur les temps et lieux où elle s'effectue.

\section{Les temps et lieux}

$\mathrm{Au}$ temps de la promulgation des actes royaux aux justiciables est associée « la volonté de donner la plus large publicité possible aux actes normatifs », laquelle «justifie le choix réfléchi du moment et de la périodicité de la publication $\gg \underline{55}$. Les indications précises sur la date de proclamation publique de patentes au vu des sources sont l'exception. Le contexte de cette formalité dans le ressort du parlement de Bordeaux avant 1547 doit être supposé plus qu'il n'est attesté.

En l'espèce, ce temps de la publication ne semble pas être celui des jours fériés, ni même celui du dimanche (lat. dominus Dei) jour de messe et d'inactivité lucrative. Au contraire, il est celui qui permet la réunion d'un maximum de justiciables et correspond par conséquent davantage aux heures et jours de marchés, lesquels sont synonymes de commerce et surtout d'affluence hebdomadaires. À l'instar des significations judiciaires, qui supposaient à la fois audiences et auditoires, les proclamations populaires avaient pour espace comme pour temps un unique marché. Présente dans quelques mandements royaux de publier, cette référence n'est pas sans rappeler la tradition romaine de diffusion de la loi puisque cette dernière était affichée " pendant trois marchés consécutifs » et c'est précisément «le lendemain du troisième jour de marché » $\underline{56}$ que lecture en était faite en présence du peuple assemblé.

Il est une question incidente du temps de cette promulgation des patentes : celle de sa répétition, souvent associée à l'idée d'une mauvaise diffusion des actes royaux. Au regard des registres du parlement, les ordonnances assorties de clauses qui mandent une publication « de nouvel « derechief » $\underline{58}$ sont trop peu nombreuses avant 1547 pour - trop vite et trop systématiquement pouvoir conclure à des difficultés de diffusion de la volonté royale. Cependant, c'est indéniable, «le choix d'un lieu adéquat importe autant que celui d'un moment propice » $\underline{59}$. Quand il s'agit de proclamer une ordonnance aux justiciables, les mandements royaux ne donnent guère de détails $\underline{60}$. D’une part parce que les villes, places et lieux où celle-ci doit être pratiquée sont " accoustuméz », la pratique, coutumière, ne nécessitant pas à cet égard un acte normatif particulier. Et d'autre part parce qu'il appartient aux magistrats eux-mêmes de définir les «lieux qu'il appartiendra » ou «endroictz» et lieux «que besoing sera». Quelquefois, le monarque sollicite même ses parlementaires en ces termes : " es lieux du ressort de notredite court que verez estre à faire » ou « en nostredite court et aultres lieux ou verrez estre à faire ». Du plus général au plus particulier, l'endroit de la signification aux justiciables des actes royaux désigne d'abord les circonscriptions administratives et territoriales qui constituent les provinces : les bailliages et sénéchaussées, les pays et seigneuries. Sont ensuite indiquées les agglomérations : en toutes les villes, cités et bourgs du royaume. Sans surprise, parmi les principales municipalités, Bordeaux est essentiellement désignée; mais d'autres de moindre importance peuvent être intéressées : Bergerac, Cadillac, Castillon, Langon, Libourne ou Soulac par exemple. «Par» ces villes, aux termes des clauses injonctives de publication, la lecture officielle des prescriptions royales est enfin signalée aux ports, havres et passages ou foires et marchés et autres lieux publics. Dans le silence des injonctions du monarque, quelques comptes-rendus de signification désignent enfin des carrefours, endroits 
« La publication des lois par des parlementaires de province ». L'exemple bor...

stratégiques des publications à son de trompe, ce qui est l'une des méthodes elle aussi accoutumée.

\section{Les acteurs et méthodes}

Concrètement, comment les parlementaires assument-ils ou supervisent-ils la publication des actes royaux ? Et d'abord, qui sont les responsables et agents de cette formalité de la proclamation dans les villes, places et lieux publics?

Cette fonction est plutôt dévolue à des sergents royaux. Même s'ils ont tendance à déléguer aux juges inférieurs de telles publications, les parlementaires n'en demeurent pas moins cette fois encore les responsables ou les agents. En termes de responsabilité tout d'abord, s'ils adressent des copies des lettres patentes aux officiers subalternes de leur ressort, les magistrats Bordelais en font également de même à l'égard de ceux des principales villes $\underline{61}$. Ils ne sont pas que responsables de la publication des actes royaux, ils en sont parfois même les agents car des huissiers de la compagnie sont mis à contribution, tantôt pour exécuter des significations populaires, tantôt pour y assister.

Il est remarquable que, durant le règne de François $\mathrm{I}^{\mathrm{er}}$, la pratique médiévale selon laquelle les agents royaux - désignés pour effectuer la proclamation en public d'une ordonnance - sollicitaient l'autorisation préalable des seigneurs hauts justiciers, soit révolue. Non seulement le souverain n'a plus à demander la moindre autorisation à des vassaux pour publier ses lettres où que ce soit dans le royaume, mais en outre et à l'inverse, il faut désormais demander à ses plus hauts « représentants » $\underline{62}$ en Guyenne - les parlementaires - la permission de publier quelque patente que ce soit dans leur ressort.

Si les prescriptions royales sont signifiées - au sens judiciaire du terme - par un agent royal, en principe sergent ou huissier, d'autres personnes que le menu peuple assistent à cette cérémonie de l'information. Par exemple les agents municipaux chargés de sonner les trompettes de la ville. En outre, des membres du parlement sont eux aussi parfois expressément requis pour assister - si ce n'est procéder - aux proclamations des édits.

Quelle que soit sa portée, il existe bien une «mise en scène » soigneusement orchestrée de la publication de la législation royale, laquelle implique une «équipe de la publication », qui agit selon une "conjonction du lieu, des personnes», à grand renfort (entre autres méthodes ) de «gestes » $\underline{3}$. Les manières de faire en ce qui concerne la notification de la législation royale au peuple témoigne de réelles singularités et ce, qu'elles soient orales ou écrites. La méthode orale - de la lecture publique nécessite concrètement de réunir un maximum de justiciables, aussi les méthodes employées sont-elles largement ritualisées. Au regard des livres de patentes de la compagnie, très peu d'actes royaux (une vingtaine) indiquent un mandement ou une injonction de publier à haute voix, son de trompe et cri public. En outre, des lieux désignés pour procéder à cette formalité, la plupart sont ceux «accoustuméz à faire cryz et publication » ou «cryz et proclamations ». Crier une ordonnance ne suppose pas d'éclat de voix particulier (ni de hurler celle-ci à qui veut l'entendre) comme le sens contemporain du terme pourrait le laisser croire. Dans l'ancien régime, cela désigne surtout le rituel solennel qui précède la «préconisation » ou lecture par le crieur public de l'acte royal. Car un cri public, s'il signifie une proclamation ou une publication, se fait après avoir amassé le peuple à son de trompe ou de tambour dans les places publiques et carrefours d'une ville, bourg et autres lieux à l'effet de rendre une chose notoire. Au $\mathrm{XVI}^{\mathrm{e}}$ siècle, il semble que ce sont les jurats de la ville qui ont la garde de «trompettes d'argent » 
mais que ces derniers ne peuvent pas « les refuser pour le service de la cour » souveraine quand celle-ci veut procéder à la proclamation d'un édit du roi64.

Une question se pose, qui est de savoir si la publication sert simplement à faire connaître la prescription dans ses grandes lignes, sous la forme d'un résumé bref que serait un « cri », ou si, au contraire, celle-ci donne lieu à la lecture intégrale du texte. Peut-on imaginer une ou deux heures de lecture publique à chaque carrefour ? Nicolas Offenstadt affirme que lors de la paix d'Arras de 1415 cela a effectivement eut lieu65. Mais qu'en est-il des ordonnances royales plus ordinaires ? Les mandements royaux de les publier donnent un semblant de réponse : lorsque certaines indiquent une lecture de «tout le contenu $\triangleq \underline{66}$ des lettres.

À défaut d'une telle précision, et a contrario, la proclamation de la patente ne saurait donc être in extenso, mais simplement par extraits, dans ses grandes lignes ou peut-être son dispositif. Du reste, ce qu'il importe de faire savoir, en particulier aux illettrés, ce sont essentiellement des défenses et interdictions d'une part, les peines encourues par les contrevenants en cas d'infraction d'autre part.

De manière vraisemblablement complémentaire (supplémentaire) il est une seconde méthode de publication des lettres royaux aux sujets, propre à la civilisation qu'induit l'écrit : celle de l'affiche. Toutefois, au vu des sources conservées, il faut attendre 1554 pour que les parlementaires Bordelais $\underline{67}$ permettent à un imprimeur de faire imprimer, vendre et exposer plusieurs édits. Néanmoins, même si les magistrats ne recourent que tardivement à la typographie pour faciliter la diffusion de certaines ordonnances royales, cela n'exclut pas qu'ils aient recours à des affiches ou «placards » 68 . Par exemple, en 1523, une autorisation de courir sus aux aventuriers et de les tailler en pièces (sic) est assortie d'un mandement de «icelle nottifier et proclamer à son de trompe, cry public et le sommaire et dispositif d'icelle actacher aux potz [poteaux ? portes ?] des halles, marchéz, cahues (sic) et autre lieux publicques »69. Si les parlementaires assument ainsi la responsabilité de la publication de la législation royale à l'échelle de leur ressort, là ne s'arrête pas leur rôle d'auxiliaires du pouvoir royal. En effet, si «des Edicts et autres les Parlemens ont le soin de l'exécution », cela signifie qu'ils «les publient» mais également qu'ils les «font observer $\gg \underline{70}$. Là est une seconde condition essentielle à la diffusion de la volonté du roi, dévolue aux parlementaires : veiller à son application $\underline{71}$ après en avoir diligenté la publication.

\section{Conclusion}

Lérection continue de parlements en province entre la seconde moitié du $\mathrm{XV}^{\mathrm{e}}$ siècle et la première du XVI ${ }^{\mathrm{e}}$ (Bordeaux n'est qu'un exemple parmi d'autres) pourrait être un moyen mis en œuvre par les souverains successifs pour assurer une meilleure diffusion de leur législation. Prémédité ou non, là est un des apports essentiels de l'établissement d'une cour souveraine en Guyenne, que ne doit pas occulter la fonction cardinale des parlementaires qui est celle de reddition de la justice. Pour leur ressort, ces derniers sont les premiers garants de la volonté du roi. Les plus hauts magistrats de l'ordre judiciaire sont des relais établis par le roi entre lui et ses sujets. Sans le concours de ses « amés et féaulx » présidents et conseillers, le souverain risque de voir ses lettres demeurer mortes. Gardiens de la loi royale, les parlementaires le sont dans la mesure où ils apportent leur aide et conseil à la diffusion des lettres royaux. Pour ce faire, ce sont eux qui en assument la publication, quitte à profiter de structures locales (municipales) déjà éprouvées. Ainsi les parlementaires de province servent la finalité théorique de l'ordonnance (laquelle ne se confond plus, à cette date, 
« La publication des lois par des parlementaires de province ». L'exemple bor...

avec la lex des Anciens, ni encore avec la loi des Modernes) : être applicable à tous et par tous, partout et tout le temps. Au quotidien et à leur échelle (qui est celle d'un ressort judiciaire), ils sont un instrument juridique de la politique royale sans lequel la législation du roi justicier ne pourrait atteindre tous ses justiciables. Personne, sous le règne de François $\mathrm{I}^{\mathrm{er}}$, n'était mieux placé que ses parlementaires pour garantir à la fois la promulgation et l'application de la volonté royale dans cette partie du royaume si longtemps restée sous domination anglaise.

À la question introductive « depuis quand légiférer est-ce communiquer ? » nous pouvons répondre : certes pas depuis les Temps Modernes ! Car, littéralement, l'on ne légiférait plus au début du XVI ${ }^{\mathrm{e}}$ siècle (au sens des Anciens Romains) et l'on ne communiquait pas encore (au sens des Anglais Modernes). Voilà qui permet de tempérer, par l'histoire, l'affirmation - sans doute propre à notre temps $\underline{72}$ - selon laquelle légiférer c'est communiquer.

\section{Notes}

1 Dans le vocabulaire juridique, la communication peut désigner un fait voire un droit : 1) Le fait de porter un évènement ou un élément d'information à la connaissance d'une personne déterminée, d'un groupe d'intéressés ou du public ; 2) L'entrée en relation d'un avocat avec un détenu dont il assure la défense, qui constitue un droit. Cf. Vocabulaire juridique, G. CoRNU (dir.), Paris, $8^{\mathrm{e}}$ éd., 2007, p. 185, $\mathrm{v}^{\circ}$ « Communication ».

$\underline{2}$ À J+1 : « Les lois et, lorsqu'ils sont publiés au Journal officiel de la République française, les actes administratifs entrent en vigueur à la date qu'ils fixent ou, à défaut, le lendemain de leur publication [...]. », C. civ. art. $1^{\mathrm{er}}$.

$\underline{3}$ La publication (et non la souveraineté de son auteur) donnerait à la loi sa vigueur, telle est une erreur commune qui semble faire droit. En toute exactitude juridique et classique (républicaine romaine $a b$ initio), si la loi oblige le peuple, ce n'est pas tant parce qu'elle est portée à sa connaissance a posteriori, après son établissement (le cas échéant par une «publication ») mais parce qu'elle est consentie par lui a priori, avant toute mesure de diffusion. La loi en effet, est constitution du peuple (assemblé en comices), elle est adoptée par lui : «Lex est, quod populus Romanus senatorio magistratu interrogante veluti consule constituebat » (la loi est ce que le peuple Romain constituait un magistrat sénatorial, par exemple un consul, l'interrogeant), J. Inst. 1. 2. 4. Comme telle, par définition, la loi est un acte public (voté par le peuple) davantage que publié (porté à la connaissance du peuple). Cette nuance est fondamentale.

$\underline{4}$ Nous suivons en cela l'exemple de Pomponius : «Necessarium itaque nobis videtur ipsius iuris originem atque processum demonstrare », D. 1. 2. 2. pr. (Nous croyons donc nécessaire de rappeler l'origine du droit et de suivre ses progrès).

$\underline{5}$ La tentation de l'anachronisme peut corrompre l'histoire du droit et des institutions. En toute rigueur méthodologique, l'historien du droit ne peut pas (et ne devrait pas) utiliser des concepts modernes, dans le langage qui est celui de son temps, pour analyser des réalités anciennes qui n'étaient pas ainsi qualifiées de leur temps, quand bien même ces réalités évoqueraient sérieusement le concept moderne. L'historien, dans l'idéal, devrait s'en tenir aux termes qui sont à sa disposition au temps de son étude, voire à ceux des temps antérieurs, mais non à ceux des temps postérieurs, pour ne pas risquer de commettre une «illusion rétrospective » (dixit 
J. KRYNEN). Il ne nous parait pas sérieux d'adopter une orientation épistémologique qui entend, tout en empruntant une appellation familière aux juristes d'aujourd'hui, reconsidérer les catégories traditionnelles. Les spécialistes de l'histoire « des normes » en conviennent : cette histoire se construit sur un présupposé historiquement erroné : « quitte à substituer un anachronisme à ceux [les vocables] qui prévalaient jusque là mais [c'est, se défendent-ils] pour mieux marquer les interactions tant à l'intérieur du champ juridique qu'au-delà. ». Cf. PETITRenaud S. et Rousselet-Pimont A., « [L'] Histoire des normes. L'émergence de la loi moderne », L'histoire du droit en France. Nouvelles tendances, nouveaux territoires, (dir.) J. KRYNEN et B. d'Alteroche, Paris, 2014, p. 223 et suivantes.

$\underline{6}$ Le terme est au professionnel ce que le mot est à l'amateur. Or, dans une terminologie scientifique, chaque terme coexiste avec une définition qui lui est propre, laquelle - littéralement (définition $\rightarrow$ définitif) - suppose une finitude. Rationnellement posée, scientifiquement établie par les meilleurs spécialistes, la définition (originelle et originale) d'un terme est ainsi immuable : elle engagera les spécialistes postérieurs de la discipline qui ne devront rien n'y additionner, ni rien n'y soustraire. C'est à cette sévère condition qu'ils pourront, en toute rigueur épistémologique, employer le terme à leur tour. L'étymologie est précieuse à cet égard, qui permet de faire coïncider, historiquement, le premier sens d'un terme avec, techniquement, le véritable sens dudit

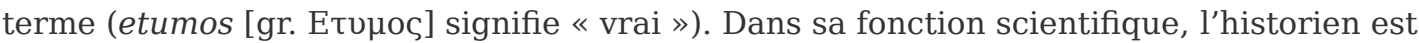
particulièrement autorisé à dater les termes qu'il emploie et à distinguer de la sorte des ères terminologiques (quatre) dans sa lexicographie. Le parlement par exemple, est un terme médiéval, dont l'usage se maintient aux temps postérieurs, modernes et contemporains. Pour autant, il est anachronique de le concevoir a priori dans l'Antiquité (ce dont ne se privent pas les constitutionnalistes actuels) et de le confondre alors avec la Boulé Athénienne ou le Sénat Romain qui s'en distinguent, historiquement et techniquement.

7 En quelque matière que ce soit et pour quelque terme technique que ce soit, l'inversion consiste à substituer une seconde définition (altérée, erronée) à une première définition (véritable), laquelle trouve son origine dans des époques précédentes (à rebours, celles du droit contemporain, du droit intermédiaire, de l'ancien droit et du droit romain).

8 «Car c'est une chose de constater l' «évolution 》 (plus exactement : d'apprécier les transformations sous le prisme de l'évolutionnisme), c'en est une autre que de changer le sens des mots. On pourra argumenter que les mots doivent refléter la réalité positive de l'époque (cette tendance a bien sûr cours chez les juristes) mais l'on se heurtera toujours au roc, pour ainsi dire " grammatical », des termes juridiques et des concepts qu'ils contiennent : les termes, "première et élémentaire dogmatique juridique » (B. Biondi) ». Cf. HeCKeTsweiler L., Populus. Éléments romains d'une restitution doctrinale de la catégorie juridique, dans Diritto@Storia, Rivista Internazionale di Scienze Giuridiche e Tradizione Romana, 2013, n 11, p. 11 [article accessible en ligne, sur le site www.dirittoestoria.it.].

9 Les fondements de la science juridique moderne sont anciens : ils sont romains, comme ceux de la science politique sont grecs. Aussi l'apport des jurisprudents romains ne doit-il pas être envisagé comme historique (et en cela daté, antique pour ne pas dire archaïque) mais technique (et en cela anhistorique).

10 LEgendRe P., L'autre Bible de l'Occident : le Monument romano-canonique. Étude sur l'architecture dogmatique des sociétés, Paris, 2009. 
« La publication des lois par des parlementaires de province ». L'exemple bor...

11 Nulle évolution (succession de temps) ne peut justifier un changement de sens : nul ne peut en volonté (ni individuelle, ni collective) changer le sens des mots sans, littéralement, les altérer (les rendre autre, en latin alter). On ne change pas le sens des mots; si nécessaire, on change de mot, quitte à concevoir un néologisme. À cet endroit, nous nous inscrivons en faux contre une partie de la doctrine qui affirme que « les concepts juridiques n'ont pas d'histoire, tout changement de sens entraînant la formation d'un nouveau concept ». Cf. TROPER M., La nécessité et le droit, Paris,

2011, p. 262. Il est pour ainsi dire schizophrénique de prétendre ainsi à un nouveau concept sur un ancien terme. Un terme (signifiant) déchu de son sens (signification) est comme une coquille vide, vidée de sa substance et livrée à l'usucapion d'un Bernard-l'ermite (alias une autre signification).

12 Pour mémoire (cf. note 3) «Lex est quod populus Romanus senatorio magistratu interrogante veluti consule constituebat », J. Inst. 1. 2. 4. Gaius en donne cette définition : "Lex est quod populus iubet atque constituit», Inst. 1, 3. (la loi est ce que le peuple «ordonne » et constitue).

13 Entre autres définitions, laquelle repose sur la dernière Constitution française en date, 1958, art. 34, Vocabulaire juridique..., p. 560-561, v « Loi ». En droit positif, la définition de la loi témoigne d'un autre problème juridique, source de confusion, qui est celui de la polysémie. Eu égard à l'enjeu de qualification qui est au cœur de la fonction du juriste, un même terme ne saurait recouvrer plusieurs sens. Aussi le scientifique est-il celui qui dispose, le cas échéant, d'autant de termes techniques que de nécessités pratiques soumises à l'appréhension de sa science. Sur ce phénomène, voir la thèse très engagée de BECQUART J., Les mots à sens multiple en droit civil français : contribution au perfectionnement du vocabulaire juridique, Lille, 1928.

14 Si elle ne fait pas l'objet d'une définition aux Institutes de Justinien (ni au Digeste), la publication y est néanmoins envisagée à trois reprises : Inst. 3. 25. 7 ; 4. 18. 4 ; 4. 18. 8 ; soit en tant que mode de dissolution du contrat de société, soit en punition de certains crimes établis par la lex Julia. Elle n'est donc pas conçue dans l'œuvre de Justinien comme une modalité législative.

15 Vocabulaire juridique..., op. cit., p. 742-743, v « Publication ».

16 Littéralement il lui faut ainsi préciser quand un autre sens est substitué à celui de principe (immédiatement ou progressivement : parfois le remplacement d'un sens par un autre se produit après un temps de coexistence des deux sens, ancien et moderne).

17 Pour une étude diachronique et juridique à la fois, voir BERRIAT SAINT PRIX J., Recherches sur les divers modes de publication des lois depuis les Romains jusqu'à nos jours, Paris, 1838.

18 Père et fils ; l'édition ici utilisée est la dernière d'Ancien Régime : de FERRIÈRE Cl.-J., Dictionnaire de droit et de pratique contenant l'explication des termes de droit, d'Ordonnances, de Coutumes \& de pratique avec les Jurisdictions de France, nouvelle édition revue et augmentée, Paris, 1771, 2 tomes.

19 Ibid., t. 2, p. 461, v « Publication».

20 Ibid., t. 2, p. 174, $\mathrm{v}^{\circ}$ «Loi ».

21 Si tant est que l'écrit soit le critère distinctif d'une civilisation. Les Institutes de Justinien précisent qu'un droit civil est établi dès lors que sont réunis une cité, des magistrats et des lois écrites : « (...) Civilia autem jura tunc esse coeperunt, cum et civitates condi, et magistratus 
creari, et leges scribi coeperunt » (Les droits civils ont commencé à être avec et la formation des cités, et la création des magistrats, et le commencement des lois écrites), J. Inst. 2. 1. 11. À cet endroit, l'erreur de traduction de Hulot (Metz, 1803) est intéressante, qui associe in fine à la loi la publication : « les lois civiles n'ont commencé d'exister que lorsqu'on a commencé à former des villes, à établir des magistrats, à publier des lois » (c'est nous qui soulignons). Sur ce thème des erreurs de traduction, voir FRÊLON E., Des erreurs de traduction aux Institutes de Justinien. Exercice de philologie juridique, dans Le droit et l'édition. Regards français et étrangers sur les mutations engagées, (dir.) J.-L. PIOTRAUT et S. EvRARD, Paris, 2016, p. 67-86.

$\underline{22}$ Ordonnance sur le fait de la Justice, août 1539, art. 110 : « Et afin qu'il n’y ait cause de douter sur l'intelligence desdits arrêts, nous voulons et ordonnons qu'ils soient faits et écrits si clairement, qu'il n'y ait ni puisse avoir aucune ambiguïté ou incertitude ne lieu à demander interprétation » et art. 111 : «Et pour ce que telles choses sont souvent advenues sur l'intelligence des mots latins contenus esdits arrests, nous voulons d'oresnavant que tous arrests, ensemble toutes autres procédures, soient de nos cours souveraines et autres subalternes et inférieures, soient de registres, enquestes, contrats, commissions, sentences, testaments, et autres quelconques, actes et exploicts de justice, ou qui en dépendent, soient prononcés, enregistrés et délivrés aux parties en langage maternel françois et non autrement. ». Au motif que « La Cour de cassation applique toujours la présente ordonnance », ces deux articles 110 et 111 sont publiés en bonne et due forme au Journal Officiel (accessibles en ligne sur le site www.legifrance.gouv.fr.).

23 Comme telle, la formule si célèbre du « langage maternel françois et non autrement » n'est pas tant, pour la France, l'acte de naissance officiel de la langue française (aujourd'hui langue « vivante ») que l'acte de décès officieux du latin (aujourd'hui langue « morte »), langue des deux droits savants de la scholastique médiévale : l'un civil (lat. ius civile) et l'autre (lat. utrumque ius) canonique.

24 Accessibles pour l'essentiel aux Archives Départementales de la Gironde (Bordeaux), série B.

25 De toutes les lettres royaux enregistrées au parlement de Bordeaux depuis sa création jusqu'en 1547, une seule fois la « loy » est mentionnée : «Loy, édit, ordonnance », ADG, 1B2, f 225, 30 avril 1517, Lettres de révocation d'aliénation du domaine. L'emploi du terme relève donc de l'exception singulière. Il peut présumer de l'interchangeabilité des trois termes (loi, édit, ordonnance), une présomption simple dans ce cas mais non irréfragable. Telle est la pratique.

26 Du moins en pratique, et si l'on relie l'acte de légiférer à l'usage nécessaire du mot loi (ou loy). En théorie, c'est depuis le Moyen Age que le Roi de France s'érige progressivement en souverain législateur, voir pour cette époque cruciale la thèse de PETIT-RENAUD S., « Faire loy »... au royaume de France de Philippe VI à Charles V (1328-1381), Paris, 2001. Si le roi de France s'avère être législateur, c'est notamment par son assimilation bien connue à l'empereur Romain. Pour ce qui est de la tradition coutumière, si typiquement médiévale, de cette assimilation des princes romains et français, voir RigaUdière A., Princeps legibus solutusest et Quod principi placuit legis habet vigorem à travers trois coutumiers du XIII ${ }^{e}$ siècle, dans Hommages à Gérard Boulvert, Nice, 1987, p. 427-451. Comme telles (comme modelées sur celles de l'empereur), les constitutions du roi ont force de loi, conformément au dogme juridique d'Ulpien : « Quod principi placuit legis habet vigorem » (Ce qui plaît au prince a la vigueur de la loi), D. 1. 4. 1. Toutefois, si les légistes médiévaux ont su utiliser l'antique plaisir du prince au profit de leurs souverains temporels, ce 
« La publication des lois par des parlementaires de province ». L'exemple bor...

n'est pas sans tronquer la formule. L'omission de « (...) utpote cum lege regia quae de imperio ejus lata est populus ei et in eum omne suum imperium et potestatem conferat » (car par la loi royale qui a établi son empire, le peuple lui a conféré l'ensemble de son empire et de sa puissance), n'est pas innocente, qui occulte l'action législative et constitutive du peuple. Sur la question du rôle juridique et politique du peuple (lat. populus), à l'époque de Justinien et du Digeste, voir la thèse de HeCKetsWeiler L., La fonction du peuple dans l'empire romain. Réponses du droit de Justinien, Paris, 2009, en particulier p. 17-72.

27 Olivier-Martin F., Les lois du roi, Cours de doctorat, Paris, 1945-1946. Cette association conçue aux Temps Modernes ne figure pas littéralement dans les sources parlementaires bordelaises antérieures au règne de Henri II.

$\underline{28}$ Une telle disparition est d'origine germanique (politique capétienne davantage que franque $a$ priori) et non romaine. Même dans l'Antiquité tardive, la réalité juridique du peuple résiste à l'omnipotence (supposée) de l'empereur romain (établi à Constantinople depuis la chute de Rome). Sur cette résistance, voir HECKETSWEILER L., La fonction du peuple..., op. cit., p. 101 et suivantes.

29 Dans la seconde moitié du XVI ${ }^{\mathrm{e}}$ siècle, un fameux théoricien de la souveraineté (entre autres auteurs, mais son influence demeure majeure) parachèvera l'altération de la république (réalité collective du peuple, somme de citoyens, lat. universi cives) en substituant à sa vraie définition celle, erronée du point de vue de la dogmatique juridique romaine, d'un « droit gouvernement de plusieurs mesnages, et de ce qui leur est commun avec puissance souveraine », J. Bodin, Les six livres de la République, Lyon, 1579, p. 1. La doctrine de l'État repose encore de nos jours largement sur l'œuvre de Bodin, sans égard pour celle de Justinien à qui l'on doit pourtant la compilation juridique la meilleure qui puisse être en termes de res publica. Sur l'altération de la République des Anciens Romains par des François Modernes, dont Bodin,voir REVERSo L., La souveraineté du peuple Romain dans la République de Jean Bodin, dans Les représentations du droit romain en Europe aux Temps Modernes, Aix-en-Provence, 2007, p. 83-99.

30 Rigaudière A., L'invention de la souveraineté, dans Pouvoirs, n 67, 1994, p. 5-20.

31 Techniquement, un peuple (populus) est défini comme une universalité des citoyens(lat. universi cives). La citoyenneté étant un attribut juridique attribué selon des conditions discriminantes : liberté, virilité, naturalité par exemple. Si le peuple est la somme des citoyens cela signifie que les personnes qui ne remplissent pas les critères de citoyenneté n'appartiennent pas, juridiquement, au peuple. Eu égard aux classifications réelles des Institutes de Justinien, la somme des citoyens et des non-citoyens dans un même espace urbain désigne une réalité non pas populaire mais universelle. Il importe à cet endroit de ne pas non plus confondre la cité (lat. civitas) et la ville, l'urbanité (lat. urbs). Cf. Les définitions des réalités extra-patrimoniales (insusceptibles d'appropriation privée) que sont les réalités communes à tous (res communes omnium), publiques (publica), universelles (universitatis), nulles (nullius), J. Inst. 2, 1, pr. à §10. L'assimilation, commune, en histoire des institutions et des idées politiques entre République et Cité romaines, Polis grecque, État moderne est vraisemblable. Est-elle rigoureusement vraie ? Les meilleurs auteurs en conviennent : la res publica médiévale (carolingienne) est « l'héritière d'une autre démarche, antérieure de près de trois siècles à la renaissance du droit romain, qui a eu pour cadre l'entourage des rois carolingiens ; une démarche que l'on peut difficilement qualifier de « juridique » stricto sensu parce que ses acteurs ont utilisé d'autres sources que les textes 
juridiques, celles que leur offraient - Cicéron, Tite-Live, Salluste et Suétone en tête - les penseurs politiques et historiens de la Rome antique. ». Cf. SASSIER Y., L'utilisation du concept de res publica » en France du Nord aux $X^{e}, X I^{e}$ et XII ${ }^{e}$ siècles, dans Structure du pouvoir, royauté et Res Publica (France, IX ${ }^{e}$-XII ${ }^{e}$ siècles), Rouen, 2004, p. 191.

32 Pour approfondir la connaissance de la lex dans la science juridique classique, et à même la source, voir Digeste, 1, 3, «De legibus... ».

33 Sur cette catégorie dogmatique qu'est le peuple voir HECKETSWEILER L., « Populus. Eléments romains... », op. cit., passim.

34 Peuple (les seuls citoyens déterminés) et public (tous indéterminés, tous ceux auxquels l'acte est opposable) ne sauraient être exactement synonymes. Cf. La définition du terme " public » en droit positif qui tient le mot «population » pour synonyme (mais non peuple) : « ensemble indéfini des individus susceptibles d'être indistinctement admis dans un lieu ou exclus de ce lieu ». Cf. Vocabulaire juridique..., $\mathrm{v}^{\circ}$ « public », p. 742.

35 «Ensemble indéfini des personnes qui peuvent être touchées par un moyen de diffusion (lecteurs, auditeurs, spectateurs, téléspectateurs) ». Cf. Ibid.

36 Littéralement cet impératif qui caractérise encore et toujours la loi est une résurgence de l'imperium antique dévolu, par les citoyens et au moyen d'une loi (la lex curiata de imperio), à quelques-uns d'entre eux : les magistrats sénatoriaux (dont est l'empereur), ceux-là même qui soumettaient au peuple les propositions de lois. Parmi les actes impératifs de l'antiquité impériale sont aussi les constitutions du prince, lesquelles conforteront des siècles plus tard (mais non, une fois encore, sans altération) le roi dans sa fonction « législative ». Il est regrettable qu'en matière de « législation » princière les romanistes empruntent, eux aussi, au droit contemporain la terminologie et la sémantique de la publication (et ce sans s'interroger sur la publicatio - in bonorum - antique). Sur ce thème des « méthodes de diffusion de la législation des Sévères », voir CORIAT J.-P., Le prince législateur, Paris, 1997, p. 603 et suivantes.

37 Une citoyenneté entendue dans un sens classique, romain et républicain, lequel suppose des conditions discriminantes dont les règles sont propres à chaque cité.

38 À cette date (règne de François $\mathrm{I}^{\mathrm{er}}$ ). Il en est quelque peu autrement aujourd'hui : la Constitution de 1958 (art. 11) renouant avec la tradition juridique de la lex, mais au moyen du néologisme (à consonance antique) de référendum. Juridiquement, la coexistence dans une même Constitution (art. 11 et art. 34 de celle de 1958) de deux modes d'élaboration de la loi (par la somme des représentants et des représentés ou par les seuls représentants) pose un problème : sauf à confondre un peuple avec un parlement, un même acte ne peut pas, institutionnellement, être à la fois décidé par tous les citoyens et par quelques-uns d'entre eux : il est l'un ou l'autre. À cet endroit, les jurisprudents classiques sont plus rigoureux, qui distinguent fort bien, parmi les sources du droit civil, la lex (constitution du peuple) du senatus-consulte (consultation du Sénat). Cf. J. Inst. 1. 2. 4 et 5.

39 La publication est au peuple, à la somme des citoyens, ce que la promulgation est au vulgaire, à la foule. Étymologiquement, la promulgatio dérive de provulgatio ou divulgatio, littéralement, ce qui est pour la foule, du vulgaire (lat. vulgus) : BERRIAT SAINT PRIX J., Recherches sur les divers modes de publication..., op cit., p. 7-8. Plus récemment, LieBs D., Promulgationslokale im 
« La publication des lois par des parlementaires de province ». L'exemple bor...

spätantiken Rom, dans Satura R. Feenstra, Fribourg, 1985, p. 215-228.

$\underline{40}$ « Divlgation n.f. (1510) est emprunté au latin divulgatio, action de répandre, révéler ». Cf. Dictionnaire historique de la langue française, A. REY (dir.), Paris, 2010, v « Divulgation », p. 666. Quant au verbe divulguer, il est « emprunté (v. 1355) au latin divulgare « rendre public » et, avec une proposition infinitive complément, « répandre le bruit que ». Ce mot est formé de dis- et vulgare « répandre dans le public, propager », « offrir à tout le monde » et en particulier « prostituer », dérivé de vulgus « commun » (vulgaire, vulgate) ». Une telle étymologie de divulguer interroge, qui confondrait vulgus et populus, vulgaire et populaire. Cf. Ibid., $\mathrm{v}^{\circ}$ «Divulguer», p. 666.

$\underline{41}$ Une fois posés en introduction nos scrupules sur l'emploi des termes de loi et de publication aux Temps Modernes, nous les maintiendrons toutefois dans le corps du texte, sans italique ni guillemets, car ils sont d'usage conventionnel en histoire des institutions.

42 Argumentaire des C@hiers du CRHiDi pour le présent numéro.

$\underline{43}$ En somme, et quitte à établir un calcul légèrement en deçà du règne de François $\mathrm{I}^{\mathrm{er}}$, depuis l'origine de la conservation des actes royaux, à partir des livres de patentes de la compagnie, 187 mandements ou injonctions peuvent être recensés, soit à peine $35 \%$ des actes royaux de quelque typologie ou contenu que ce soit enregistrés entre 1463 et 1547.

$\underline{44}$ Et qui annonce l'adage contemporain en vertu duquel «nul n'est censé ignorer la loi ». Sur ce thème, et pour l'époque médiévale susdite, consulter : K. WEIDENFELD, « Nul n'est censé ignorer la loi » devant la justice royale (XIV $-X V^{e}$ siècles), dans Information et société en Occident à la fin du Moyen Age, (dir.) C. Boudreau, K. Fianu, Cl. Gauvard, M. Hebert, Paris, 2004, p. 165-183.

$4 \underline{5} 35$ patentes seulement, soit moins de $7 \%$, de celles qui sont archivées.

46 L'expression est de Fogel M., Les cérémonies de l'information dans la France du XVI ${ }^{e}$ au milieu du XVIII ${ }^{e}$ siècle, Paris, 1989.

47 Cf. supra, note 39.

48 Cf. supra, note 38.

$\underline{49}$ Selon S. PetiT-RENAUd, « Pour toute la doctrine, il est clair qu'une loi ne peut être opposable sans avoir été publiée », ce que l'auteur s'attache à justifier à partir notamment de saint Thomas, Balde, Gilles de Rome, Gerson ou des coutumiers médiévaux. Cf. PETIT-RENAUd S., « Faire loy »..., op. cit., p. 396-398.

$\underline{50}$ Cf. supra, note 26.

$\underline{51}$ À ceci près que le peuple, librement (la liberté, antonyme de la servitude, est un critère de citoyenneté), n'a pas à être régi par un roi s'il se gouverne lui-même, la promulgation des prescriptions royales n'induit pas que des contraintes puisque, les préambules le rappellent, c'est avant tout le bien de ses gouvernés (les sujets) qui justifie l'action du gouvernant (le roi). Cf. BABEAU A, Les préambules des ordonnances royales et l'opinion publique, Paris, 1896. Plus récemment mais pour une période postérieure à notre champ d'étude, voir la thèse de SEIGNALET- 
MAUHourat F, «A ces causes... » Essai sur les préambules des ordonnances royales au XVII et XVIII ${ }^{e}$ siècles, Toulouse I, 2004.

52 Pour l'époque médiévale, voir Lett D. et Offenstadt N. (dir.), Haro ! Noel ! Oyé ! Pratiques du cri au Moyen-Age, Paris, 2003.

53 AM Bordeaux, Ms 760, fำ 979, 28 mai 1543.

54 AM Bordeaux, Ms 760, fo 746, 30 août 1541.

55 Petit-Renaud S., « Faire loy »..., op. cit., p. 418.

56 Berriat SAINT PRIX J., Recherches sur les divers modes de publication de publication..., op. cit., p. 8 .

$\underline{57}$ ADG, 1B3, fo 293, 14 juillet 1536.

58 Ibid., f $117 \mathrm{v}^{\circ}, 18$ mars 1528 n. s. ; ADG, 1B4, fo $85 \mathrm{v}^{\circ}, 27$ mai 1540.

59 CAUChies J.-M., La législation princière dans le comté de Hainaut, ducs de Bourgogne et premiers Habsbourg (1427-1506), Bruxelles, 1982. Bien des conclusions de cette thèse remarquable sont applicables à d'autres princes, en particulier aux rois de France de la fin du Moyen Age et du début des Temps Modernes.

60 La référence exacte des mentions de ces détails est dans notre thèse : FRÊLON E., Le Parlement de Bordeaux et la «loi »(1451-1547), Paris, 2011, p. 513-514.

61 Ceci est attesté par la présence dans des archives municipales (celles d’Agen par exemple) de lettres royaux sur le repli desquelles est écrit « Donné par copie », suivi de la signature du greffier civil et criminel de la cour « De Pontac » : AM Agen, CC 49, f 77 vº̀ $^{\circ}$, Copie de lettres autorisant, à la requête des gens des États de Guyenne, le commerce avec l'Espagne, $1^{\text {er }}$ juillet 1528 ; mentionnée comme n³044 du Catalogue des actes de François $I^{\text {er }}$, t. 1, Paris, 1887 et $n^{\circ} 488$ des Ordonnances des Rois de France, Règne de François I ${ }^{e r}$, t. 5, Paris, 1936.

62 KRYNEN J., Qu'est-ce qu'un Parlement qui « représente le roi »?, dans Excerptiones iuris : Studies in Honor of André Gouron, Berkeley, 2000, p. 353-366 ; ID. De la représentation à la dépossession du roi : les parlementaires "prêtres de la justice », dans La représentation dans la tradition du « ius civile » en Occident, Rome, 2002, p. 95-119 ; FRÊLON E, Les compétences extrajudiciaires du Parlement de Bordeaux (XVe-XVIII siècles), dans Le Parlement de Bordeaux (1462-2012). 550 ans d'histoire du Parlement et du Barreau de Bordeaux, (dir.) B. FAVREAU, Bordeaux, 2014, p. 53-66.

63 Offenstadt N., Annoncer la paix. Publication et sujétion pendant la guerre de Cent ans, dans Cahiers d'histoire, $\mathrm{n}^{\circ}$ 66, 1997, p. 23-36 ; ID., Les crieurs publics à la fin du Moyen-Âge, dans Information et société..., op. cit., p. 203-217.

64 Sous le règne non de François $\mathrm{I}^{\mathrm{er}}$ mais de son fils Henri II, « La cour remonstra à Poncastel, jurat de cette ville, que, quand il a fallu faire la publication de l'Edit du Roy qui porte deffenses de porter des armes à feu que les jurats n'ont vouleu bailler les trompettes d'argent, s'excusant 
«La publication des lois par des parlementaires de province ». L'exemple bor...

qu'elles estoient rompues et ce que ledit de Poncastel a assuré estre vray par ce ladite cour enjoint aux jurats de les faire accoustrer au plus tost et ne les refuser pour le service de la cour ». Cf. AMB, FF2, fo 1, 1557.

65 OffenstadT N., Les crieurs publics..., art. cit., passim.

66 Pour des références détaillées voir : FRÊLON E., Le Parlement de Bordeaux..., op. cit., p. 530, note 284 .

$\underline{67}$ Le retard ainsi accusé d'une cour de province sur le Parlement de Paris est de plus d'un demisiècle : « dès l'apparition de l'imprimerie on recourut à ce procédé [...] Tel est le cas du Parlement de Paris en mars 1500 ». Cf. ESMONIN E., La publication et l'impression des ordonnances royales sous l'Ancien Régime, dans Études sur la France des XVII et XVIII ${ }^{e}$ siècles, Paris, 1964, p. 179.

68 Les archives à disposition, pour la période envisagée, ne renferment pas d'exemplaires d'ordonnances publiées en Guyenne sous forme de petits livres ou pamphlets, comme c'était par exemple le cas aux Pays Bas.

$\underline{69}$ ADG, 1B2, fo 445, 25 sept. 1523 .

70 DE LA Roche Flavin B., Treze livres des parlemens de France, Bordeaux, 1617, liv. XIII, p. 707.

71 Pour une étude de l'application de la « législation » royale par le parlement de Bordeaux entre 1451 et 1547, voir : FRÊLON E., Le Parlement de Bordeaux..., op. cit., p. 533-568.

$\underline{72}$ Aujourd'hui sans doute ; mais non hier, du moins non dans la plus haute Antiquité « républicaine », quand légiférer c'était, pour le peuple, constituer tandis que publier était attribuer à ce même peuple, en son entier, les biens de l'un des citoyens dont il était composé. La question reste donc entière : depuis quand légiférer est-ce communiquer ? Dans la quête de cette origine, la « Renaissance » est un jalon crucial, qui ruine la civilisation latine afin de mieux établir la civilisation française.

PDF généré automatiquement le 2020-07-11 04:21:56

Url de l'article : https://popups.uliege.be:443/1370-2262/index.php?id=424 